\title{
Fault Diagnosis of Subsea Blowout Preventer Based on Artificial Neural Networks
}

\author{
Zengkai Liu ${ }^{1}$, Yonghong Liu ${ }^{1}$, Baoping $\mathrm{Cai}^{1}$, \\ Dawei Zhang ${ }^{1}$ and Junlei $\mathrm{Li}^{2}$ \\ ${ }^{1}$ College of Mechanical and Electronic Engineering, \\ China University of Petroleum, China \\ ${ }^{2}$ Wanhua Chemical Group Co., Ltd
}

\begin{abstract}
Subsea blowout preventer is an important tool for ensuring safety of drilling activities and rig personal. In case of faults, it might cause severe damages to the environment and oil companies. This paper presents the method to perform fault diagnosis of subsea blowout preventer (BOP) based on artificial neural network (ANN).BP ANN of the BOP are proposed on the basis of the typical faults of the BOP in the process of opening and closing. In order to obtain higher training speed and precision, BP ANN are improved with gradient descent with momentum and adaptive LR gradient descent methods. Besides, RBF network is also presented for getting a better model for diagnosis. Compared with BP network, RBF network has better performance concerning training speed and precision in this case. However, BP network will show stronger flexibility in the complex model with plenty of fault types.
\end{abstract}

Keywords: subsea blowout preventer, artificial neural network, fault diagnosis

\section{Introduction}

Neural network or artificial neural network (ANN) refers to the nonlinear system made up of lots of computing elements (neurons), and it imitates information processing, storage and retrieval functions of human brain systems in a certain degree. Thus, it has the intelligent processing functions such as learning, memory and computing [1]. At present, there are more than 40 kinds of neural networks and some typical neural networks are BP network, the Hopfield net, CMAC cerebellum model, radial basis function (RBF) network and ELMAN network, et al. These neural networks have good application prospect in pattern recognition, system identification, economics, biology, medicine, signal processing, automatic control, fault diagnosis, combinatorial optimization, forecast and other fields, and its good intelligence features can solve many of practical problems difficult to modern computers.

BP neural network is a multilayer feed-forward network which is the most widely until now [2]. It uses Error Back-propagation algorithm, namely BP algorithm. Generally, the feedforward network with a single hidden layer is called "three-layered feed-forward network", consisting of input layer, hidden layer and output layer. Figure 1 is a three-layered BP network model diagram. Where, the input vector is $P=\left(\mathrm{X}_{1}, \mathrm{X}_{2}, \ldots, \mathrm{X}_{\mathrm{i}}, \ldots, \mathrm{X}_{\mathrm{n}}\right)^{T}$ and the hidden layer's output vector is $Y=\left(\mathrm{Y}_{\mathrm{P}} \mathrm{Y} \square_{2}, \ldots, \mathrm{Y}_{\mathrm{i}}, \ldots, \mathrm{Y}_{\mathrm{m}}\right)^{T}$. The output layer's output vector is $O=\left(\mathrm{O} \sqcap \mathrm{O}_{2}, \ldots, \mathrm{O}_{\mathrm{k}}, \ldots, \mathrm{O} \varphi^{T}\right.$ and the desired output vector $d=\left(\mathrm{d}_{\Gamma_{\mathrm{C}}} \mathrm{d}_{2}, \ldots, \mathrm{d}_{\mathrm{k}}, \ldots, \mathrm{d}_{1}\right)^{T}$. Besides, the weight matrix from the input layer to the hidden layer is denoted by $V=\left(\mathrm{V}_{\mathrm{T}} \mathrm{V}_{2}, \ldots, \mathrm{V}_{\mathrm{j}}, \ldots, \mathrm{V}_{\mathrm{m}}\right)^{T}$, wherein the column vector $\mathrm{V}_{\mathrm{j}}$ is the weight vector corresponding 
to the $\mathrm{j}$-th hidden layer neuron and the weight matrix from the hidden layer to the output layer is represented by $W=\left(\mathrm{W}_{\mathrm{T}} \mathrm{W}_{2}, \ldots, \mathrm{W}_{\mathrm{k}}, \ldots, \mathrm{W}_{1}\right)^{T}$, wherein the column vector $\mathrm{W}_{\mathrm{k}}$ is the weight vector corresponding to the $\mathrm{k}$ th output layer neuron. The learning rules process of $\mathrm{BP}$ network includes the following steps: (1) Initialization; (2) Input the training samples first and then calculate the output of every layer; (3) Calculate the network output error; (4) Calculate the error signal of every layer; (5) Adjust the weight value; (6) Check whether all the samples have been completed trained in rotation at a time; (7) Check whether the network's total error is to meet the requirement of accuracy.

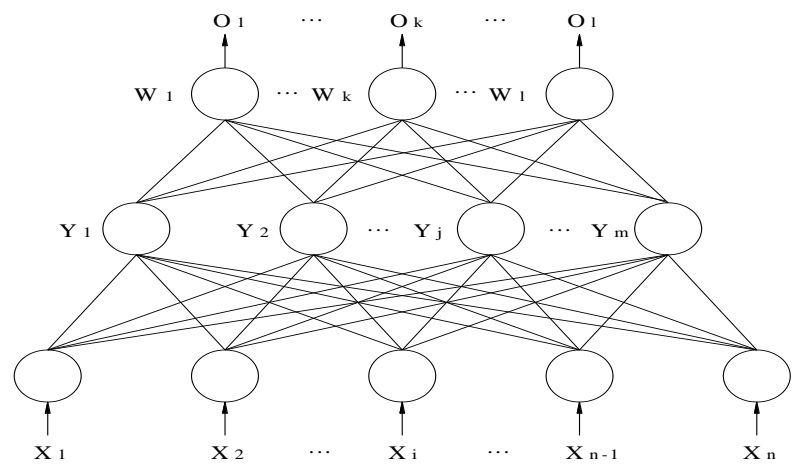

Figure 1. Schematic Diagram of Three-layered BP ANN

A classic RBF network consists of input layer, one hidden layer and an output layer. The characteristics of the RBF network are as follows. RBF is a single hidden layer and the weight from input layer to hidden layer is fixed to 1. It has "local mapping" characteristics, which is a kind of neural network with local response characteristics. The nonlinear transformation's effect of the hidden nodes of RBF network is similar to BP network' and both of them are converting the linear inseparable problem into linear separable problem [3].

ANN is widely used in various fields such as hydrological problems, multiple cracks detection, face recognition, wind speed modeling et al., [4-7]. Many researchers have presented diagnostic methods by using ANNs to detect the problems of rotating machine parts [8], check-valves [9], hydraulic systems [10], pumps [11] and gas turbines [12].

This paper aims to combine the knowledge about ANN with the fault diagnosis of the subsea blowout preventer. The BP or RBF networks are established by MATLAB software to calculate and diagnose the extracted data so that the fault diagnosis of BOP can be carried out.

\section{Typical Fault Analysis of Subsea BOP}

This paper analyzes several typical faults of the BOP in the process of opening and closing due to the limitation of experimental conditions. Pressure waveform data of important motoring sites can be obtained by installing sensors in the hydraulic circuit. Then the required fault data can be extracted through the treatment of pressure waveform of the sensitive point.

Hydraulic circuit of ram BOP is shown in Figure 2. Three monitoring sites are set for obtaining the pressure data. The monitoring site A can detect the pressure at the entrance when the ram is closed. The monitoring site B can detect the pressure of entrance when the ram is open. The monitoring point $\mathrm{C}$ is placed on the inner wall of the ram and shell. So when the ram is closed, the enclosed space is formed between the shell wall and pipe wall. 
Therefore, the monitoring $\mathrm{C}$ can detect if there is an internal leakage (through the closed ram).

This paper mainly aims to analyze four typical faults, namely "failed to close", "internal leakage (through the closed ram)", "failed to open", and "failed to fully open". Real-time monitoring of pressure in the circuit is realized by installing three sensors A, B and C. So the changes of three pressure waveforms can be obtained through the feedback signal of the sensors.

Characteristic values of the pressure waveforms can be used to determine whether the pressure reaches the expected value in a specific period. Output vector $\left(\begin{array}{lllll}0 & 0 & 0 & 0 & 1\end{array}\right)$ means the BOP is normal operation. ( $\left(\begin{array}{lllll}0 & 0 & 0 & 1 & 0\end{array}\right)$ denotes that the BOP is failed to close. $\left(\begin{array}{lllll}0 & 0 & 1 & 0 & 0\end{array}\right)$ shows

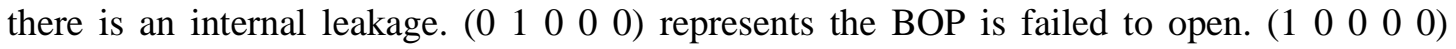
denotes the BOP is failed to fully open.

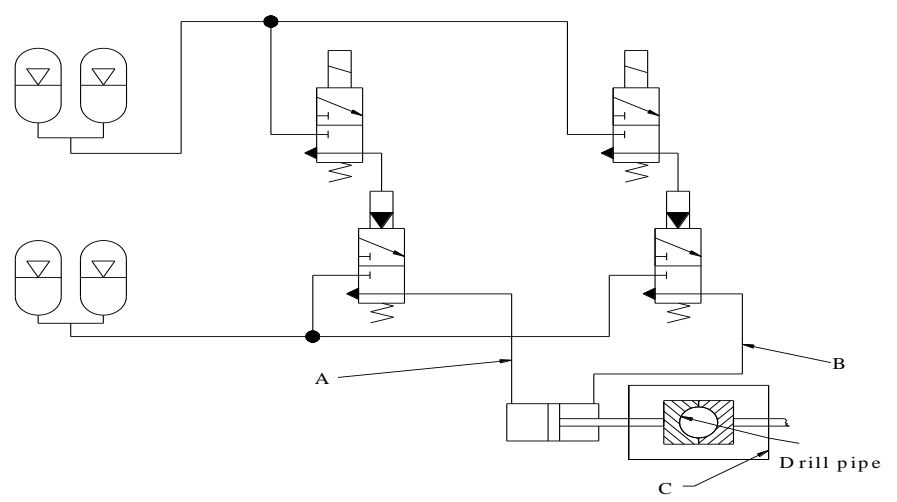

Figure 2. Hydraulic Circuit Diagram of Deepwater BOP

\section{Fault Diagnosis based on Neural Network}

Artificial neural network is to simulate the human's brain to realize training and learning process. Figure 3 is the structure of fault diagnosis system based on ANN.

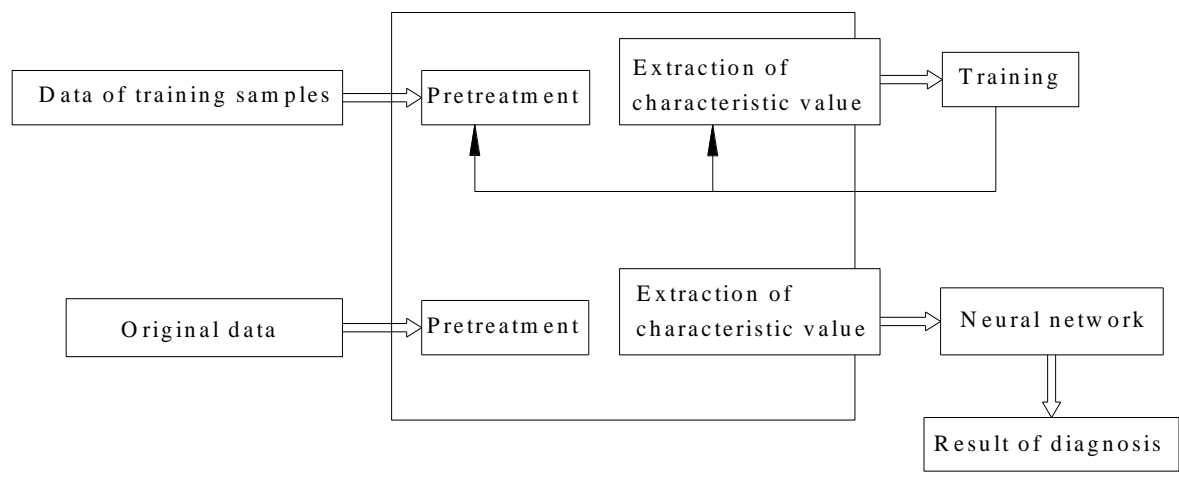

Figure 3. General Structure of Fault Diagnosis System based on ANN

\subsection{BP Network}

Firstly, data preprocessing is performed. As Sigmoid function is often used for hidden layers in BP network, the input value is required between 0 and 1 to improve the training rate 
and sensitivity. Normalization of input data is realized by using Premnmx function and the data distribution is in interval $[-1,1]$.Secondly, select and set the parameters.

(1) Determine the number of hidden layers. In general, the network error can be reduced by increasing the number of hidden layers. As there are not much training data, a BP network with two hidden layer is constructed to minimize the error.

(2) The number of hidden layer neurons is determined by the empirical Equation 1.

$$
\mathrm{y}=\sqrt{\mathrm{m}+\mathrm{n}}+\mathrm{a}
$$

Where, $y$ is the number of hidden layer neurons; $n$ is the number of input layer neurons; $\mathrm{m}$ is the number of output layer neurons; $a$ is a constant between 0 and 10 .

In this paper, the number of input layer neurons and output neurons is set as 12 and 5, respectively. Therefore, the number of hidden layer neurons can be selected from 4 to 14 . Eventually, after a lot of trainings and validation, the number of two hidden layer neurons is selected as 12 and 9, respectively.

(3) The other parameters are determined as follows: Training iterative process: net. TrainParam. Show = 25; Vector: $n e t$. TrainParam. $L r=0.02$; Maximum number of training: net. TrainParam. Epochs $=20000$; Training precision: $n e t$. TrainParam. Goal $=1 e-3$. In this way, a complete BP network can be established by using the newff function in MATLAB after determining all the parameters.

TRAINGDM algorithm is utilized for training and the key statement in the program is [net, $t r]=$ train $(n e t, P, T)$. The training of BP network will randomly generate a set of initial weights and threshold values each time, and the required accuracy can be obtained through the constant adjustment of these values. Therefore, the initial weights and threshold values will directly affect the speed of training, so a reasonable network must be chosen after lots of repeated trainings.

The convergence curve of training process is shown in Figure 4. It is shown in the figure that the error precision of BP network reaches 0.001 after 18262 times of trainings. It needs too much training and time. Therefore, the network needs to be improved.

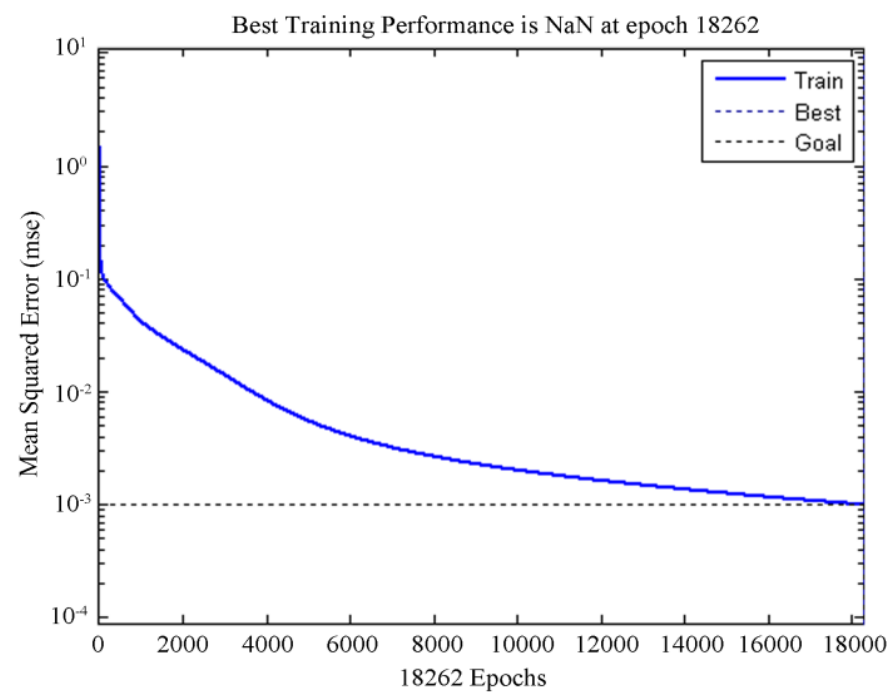

Figure 4. Convergence Curve of BP Network Training Process 
Three samples of data as the input are used to test the built network. Before the test, these data must be preprocessed. The output values of BP network and the expected results are listed in Table 1 as follows. Although there are some errors, the test results are close to the expected results. It shows that the proposed BP network can perform fault diagnosis for subsea BOP.

Table 1. Output Values of BP Network and the Expected Results

\begin{tabular}{|c|c|c|}
\hline Samples & Output results of BP network & Expected results \\
\hline 1 & $(-0.0264-0.00070 .0179-0.00451 .0066)$ & $\left(\begin{array}{lllll}0 & 0 & 0 & 0 & 1\end{array}\right)$ \\
\hline 2 & $(0.0123-0.01340 .00040 .9847-0.0106)$ & $\left(\begin{array}{lllll}0 & 0 & 0 & 1 & 0\end{array}\right)$ \\
\hline 3 & 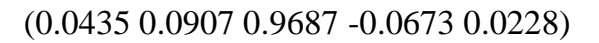 & $\left(\begin{array}{llllllllll}0 & 0 & 1 & 0 & 0\end{array}\right)$ \\
\hline
\end{tabular}

\subsection{The Improvement of BP Network}

A three-layered neural network is established to be the comparison object, taking 0.02 as learning rate and 0.01 as the training precision. Other parameters are similar to the former network. The network can be improved by gradient descent with momentum (GDM). Function traingdm is used for this method, which can update weight and bias values according to GDM. The main MATLAB statement for improvement is "net. TrainParam.MC $=0.98^{\prime \prime}$, which means the momentum factor is 0.98 . Figure 5 and 6 respectively show the convergence curves before and after improving the network training process.

The standard BP network achieves the expected accuracy after 42 seconds and 5448 times of trainings. However, the improved BP network can achieve the same accuracy after only 26 seconds and 3829 times of trainings with a distinct advantage. The same samples in Table 1 are used to test the improved BP network. The results are listed in Table 2. For the improved BP network based on GDM, it has similar errors like the standard BP network. But the improved BP network has faster training speed.

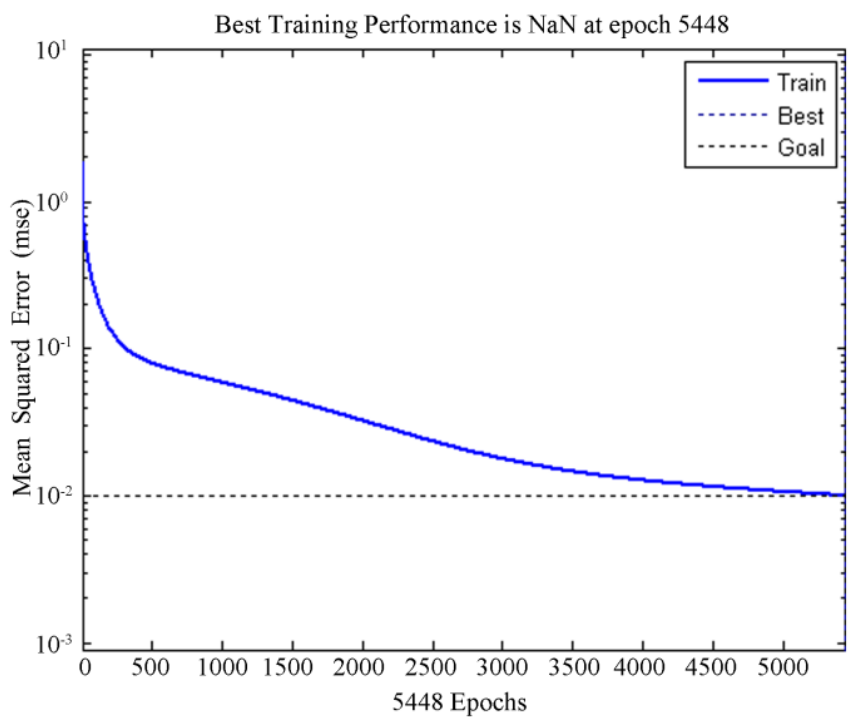

Figure 5. Convergence Curve of Standard BP Network Training process 


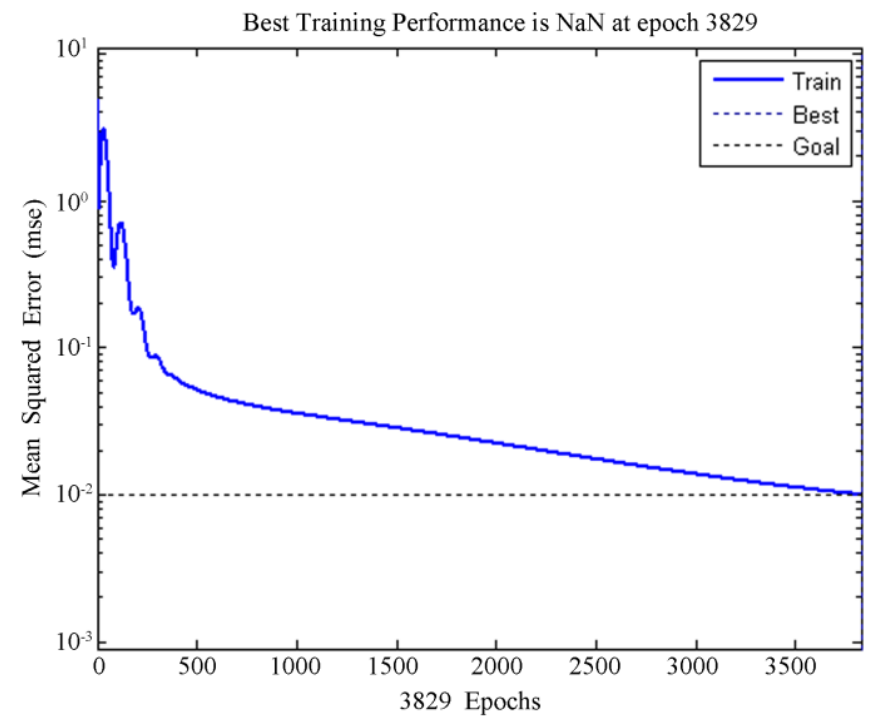

Figure 6. Convergence Curve of the Improved BP Network based on GDM

Table 2. Test Results of the Improved BP Network based on GDM

\begin{tabular}{|c|c|c|c|}
\hline Samples & Output of standard BP network & $\begin{array}{c}\text { Output of the improved BP } \\
\text { network based on GDM }\end{array}$ & $\begin{array}{l}\text { Expected } \\
\text { results }\end{array}$ \\
\hline 1 & $\begin{array}{c}(-0.02770 .08080 .05180 .1572 \\
0.8251)\end{array}$ & $\begin{array}{c}(0.11190 .07640 .10740 .2078 \\
0.8240)\end{array}$ & $\left(\begin{array}{lllll}0 & 0 & 0 & 0 & 1\end{array}\right)$ \\
\hline 2 & $\begin{array}{c}(0.0092-0.00010 .00581 .0443 \\
-0.0718)\end{array}$ & $\begin{array}{c}(0.0513-0.1202-0.03731 .0757- \\
0.0308)\end{array}$ & $\left(\begin{array}{lllllll}0 & 0 & 0 & 1 & 0\end{array}\right)$ \\
\hline 3 & 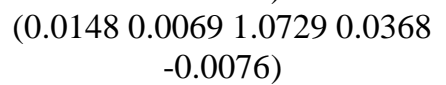 & $\begin{array}{c}(0.0309-0.02420 .9576-0.0194 \\
0.0215)\end{array}$ & $\left(\begin{array}{lllll}0 & 0 & 1 & 0 & 0\end{array}\right)$ \\
\hline
\end{tabular}

Learning rate (LR) has great effects on the network using BP algorithm or gradient descent method with momentum. Adaptive LR gradient descend (ALRGD) can improve BP network by adaptively changing LR to increase its stability and speed. The ALRGD method can be implemented on the basis of the original three-layered BP network, using the traingda function. The training process is shown in Figure 7. The standard BP network can achieve the required accuracy after 42 seconds and 5448 times of trainings, while the improved network with ALRGD needs 14 seconds and 449 times of trainings to reach the same accuracy. For testing the improved network, the same samples are adopted shown in Table 3. It demonstrates that the improved network has slightly smaller errors and higher training speed.

Table 3. Test Results of the Improved BP Network based on ALRGD

\begin{tabular}{|c|c|c|c|}
\hline Samples & $\begin{array}{c}\text { Output of the standard BP } \\
\text { network }\end{array}$ & $\begin{array}{c}\text { Output of BP network based } \\
\text { on ALRGD }\end{array}$ & $\begin{array}{l}\text { Expected } \\
\text { results }\end{array}$ \\
\hline 1 & $\begin{array}{c}(-0.02770 .08080 .05180 .1572 \\
0.8251)\end{array}$ & $\begin{array}{c}(0.05760 .02180 .02150 .1383 \\
0.7655)\end{array}$ & $\left(\begin{array}{llllll}0 & 0 & 0 & 0 & 1\end{array}\right)$ \\
\hline 2 & $\begin{array}{c}0.0092-0.00010 .00581 .0443 \\
-0.0718)\end{array}$ & $\begin{array}{c}0.1065-0.05470 .02971 .0214 \\
-0.1613)\end{array}$ & $\left(\begin{array}{lllll}0 & 0 & 0 & 1 & 0\end{array}\right)$ \\
\hline 3 & $\begin{array}{c}(0.01480 .00691 .07290 .0368 \\
-0.0076)\end{array}$ & $\begin{array}{c}(0.1152-0.00060 .9763-0.1661 \\
-0.0138)\end{array}$ & $\left(\begin{array}{lllll}0 & 0 & 1 & 0 & 0\end{array}\right)$ \\
\hline
\end{tabular}




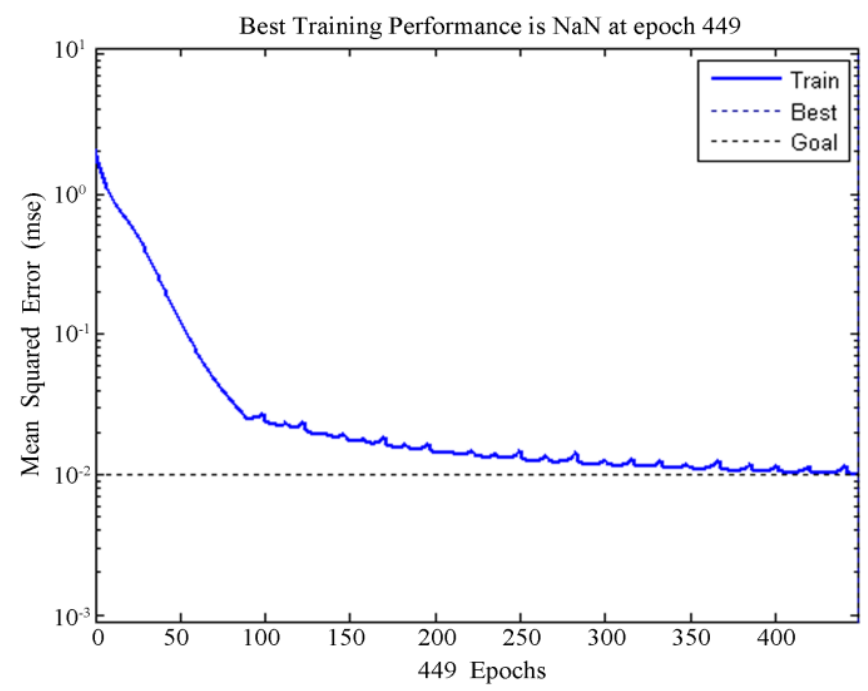

Figure 7. Convergence Curve of the Improved BP Network based on ALRGD

\subsection{RBF Network}

The parameters will directly affect the training speed and final accuracy. For the sake of comparing the BP network and the RBF network, the target error is set as 0.001 . The convergence process during the training is shown in Figure 8. It shows that only 11 times of trainings are needed to achieve the expected error precision. Obviously, RBF network has faster training speed than BP network. The same samples in Table 1 are used to test the proposed RBF network. The test results and the expected results are listed in Table 4.

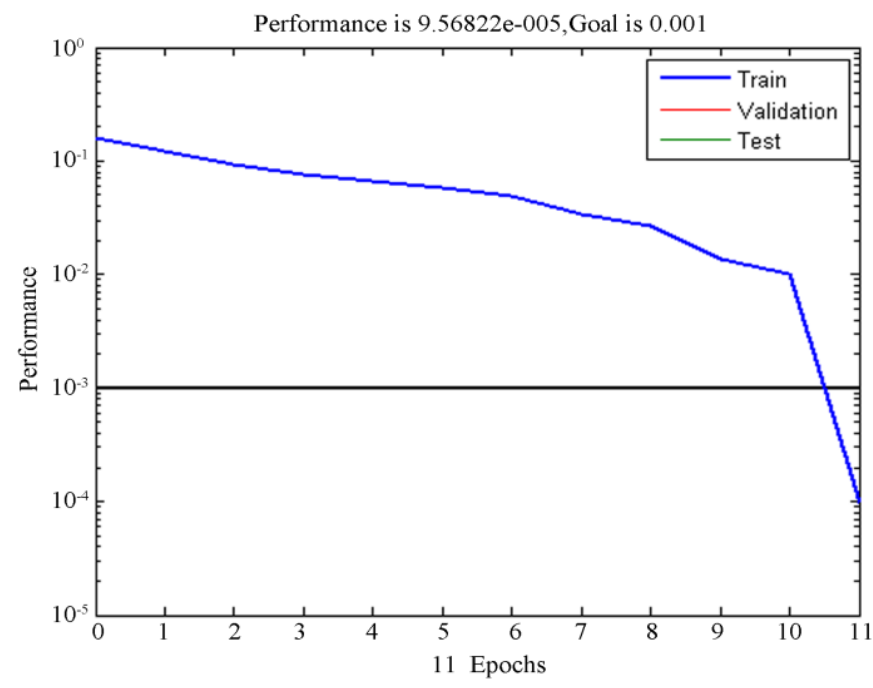

Figure 8. Convergence Curve of RBF Network

BP network and RBF network have different characteristics. On the one hand, BP network has slow learning speed, because its weight can't be calculated directly and it needs constant adjustment to achieve the final requirements. RBF network has only one hidden layer and the weight can be calculated directly, where the weight adjustment calculation is not needed and 
a lot of time is saved. Besides, BP network is sensitive to some parameters such as learning rate. On the other hand, RBF network has higher calculation precision and lower error value than BP network. However, there are not many fault types are researched in this paper, so RBF network performs better than BP network in general. If there are plenty of actual fault types, BP network will show stronger ability to adapt in the complex model.

Table 4. Test Results of the RBF Network

\begin{tabular}{|c|c|c|}
\hline Samples & Output of the RBF network & Expected results \\
\hline 1 & $(0.0000-0.00560 .00000 .00051 .0051)$ & $\left(\begin{array}{lllll}0 & 0 & 0 & 0 & 1\end{array}\right)$ \\
\hline 2 & $(0.0000-0.03590 .00001 .0359-0.0000)$ & $\left(\begin{array}{lllll}0 & 0 & 0 & 1 & 0\end{array}\right)$ \\
\hline 3 & $(-0.00000 .06020 .9395-0.00000 .0003)$ & 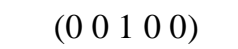 \\
\hline
\end{tabular}

\section{Conclusions}

This paper presents the method for fault diagnosis of subsea BOP based on ANN. To improve the performance of the network, different algorithms of ANN are studied.

(1) ANN can be used for fault diagnosis of the BOP. The fault type will be obtained based on the input data of the BOP in the proposed BP, improved BP and RBF networks. Some samples are used to test the developed networks.

(2) The improved BP networks with GDM or ALRGD have higher training speed and precision to a certain extent.

(3) BP and RBF networks have their own advantages and disadvantages. However, RBF network is better than BP network in the case that there are not many fault types to be diagnosed.

In the future research work, the methodology to determine the optimum number of hidden layers neurons for the network will be presented. Besides, how to optimize the learning samples and make the diagnosis results more accurate will be addressed.

\section{Acknowledgements}

The authors wish to acknowledge the financial support of the National High-Technology Research and Development Program of China (2013AA09A220), Program for Changjiang Scholars and Innovative Research Team in University (IRT1086), the Fundamental Research Funds for the Central Universities (14CX06052A), the Innovation Project Foundation of Graduate School of China University of Petroleum (CX2013054) and the Fundamental Research Funds for the Central Universities (13CX02077A), National Natural Science Foundation of China (51309240), Ph.D. Programs Foundation of Ministry of Education of China (20130133120007), and Incubation Program of Excellent Doctoral Dissertation of China University of Petroleum (No. 2013-4).

\section{References}

[1] M. Demetgul, I. N. Tansel and S. Taskin, "Fault diagnosis of pneumatic systems with artificial neural network algorithms", Expert Sys Appl, vol. 36, (2009).

[2] M. Karpenko, N. Sepehri and D. Scuse, "Diagnosis of process valve actuator faults using a multilayer neural network, Control Eng. Pract., vol. 11, (2003).

[3] A. G. Parlos, K. Kim and R. M. Bharadwaj, "Sensorless detection of mechanical faults in electromechanical systems", Mechatronics, vol. 14, (2004). 
[4] D. Bisht, S. Jain and M. M. Raju, "Prediction of water table elevation fluctuation through fuzzy logic and artificial neural networks", Int J Adv Sci and Technol, vol. 51, (2013).

[5] P. R. Baviskar and V. B. Tungikar, "Multiple cracks assessment using natural frequency measurement and prediction of crack properties by artificial neural network", Int J Adv Sci and Technol, vol. 54, (2013).

[6] K. L. Reddy, G. R. Babu and L. Kishore, "Face recognition based on eigen features of multi scaled face components and artificial neural network, Int. J. Secur. Appl., vol. 5, (2011).

[7] J. Lee, G. L. Park, E. H. Kim, Y. Kim and I. W. Lee, "Wind speed modeling based on artificial neural networks for Jeju Area", Int. J. Control Autom., vol. 5, (2012).

[8] S. Rajakarunakaran, P.Venkumar, D. Devaraj and K. S. P. Rao, "Artificial neural network approach for fault detection in rotary system", Appl. Soft Comput. J., vol. 8, (2008).

[9] S. H. Seong, S. Hur, J. S. Kim, J. T. Kim, W. M. Park, U. C. Lee and S. K. Lee, "Development of diagnosis algorithm for the check valve with spectral estimations and neural network models using acoustic signals", Ann Nucl Energy, vol. 32, (2005).

[10] M. Demetgul, "Experiment based fault diagnosis on bottle filling plant with LVQ artificial neural network algorithm", Pamukkale Univ J Eng Sci, vol. 14, (2008).

[11] M. A. Karkoub, O. E. Gad and M. G. Rabie, "Predicting axial piston pump performance using neural network", Mech Mach Theory, vol. 34, (1999).

[12] M. Karpenko and N. Sepehri, "Neural network classifiers applied to condition monitoring of a pneumatic process valve actuator", Eng Appl Artif Intell, vol. 15, no. 3-4, (2002). 
International Journal of Security and Its Applications Vol.8, No.2 (2014) 